\title{
Continuous positive airway pressure titration for obstructive sleep apnoea: automatic versus manual titration
}

\author{
Nigel McArdle, ${ }^{1}$ Bhajan Singh, ${ }^{2}$ Michelle Murphy, ${ }^{2}$ Kevin R Gain, ${ }^{1}$ Christine Maguire, ${ }^{2}$ \\ Sarah Mutch, ${ }^{2}$ David R Hillman ${ }^{2}$
}

- Supplementary tables are published online only. To view these files please visit the journal online (http://throax.bmj. com)

${ }^{1}$ University of Western Australia, School of Medicine and Pharmacology, Respiratory Medicine Department, Royal Perth Hospital, Western Australia

${ }^{2}$ Sir Charles Gairdner Hospital: Western Australian Sleep Disorders Research Institute Department of Pulmonary Physiology, Perth, Western Australia

Correspondence to Dr Nigel McArdle, University of Western Australia, School of Medicine and Pharmacology, Respiratory Medicine Department, Royal Perth Hospital, Perth 6000, Western Australia;

nmcardle@cyllene.uwa.edu

Received 5 April 2009 Accepted 9 April 2010

\begin{abstract}
Background and aims Manual laboratory continuous positive airway pressure (CPAP) titration for obstructive sleep apnoea (OSA) is costly, time intensive and delays access to treatment. Automatic positive airway pressure (APAP) titration has the potential to reduce cost and improve access to treatment. The aim of this study was to assess the clinical efficacy and costs of APAP titration compared with manual titration in moderate-severe OSA. Methods Patients with moderate-severe OSA (apnoea/ hypopnoea index $>15$ and Epworth Sleepiness Score $\geq 8$ ) who were free of co-morbidities that could impair APAP titration were eligible. 249 participants were randomised to manual titration, home APAP or laboratory APAP titration to determine a fixed pressure for CPAP. Clinical and direct cost outcomes were assessed after 4 weeks of treatment.
\end{abstract}

Results Average nightly CPAP use, subjective sleepiness, SF36 quality of life, Trails A and B cognitive function and polysomnographic outcomes were similar among the per-protocol groups. Non-hypertensive patients had a lower resting heart rate (and greater reduction in heart rate) at 4 weeks after laboratory APAP titration compared with home APAP titration. Costs per patient were highest in manual (AU\$817.84), followed by laboratory (AU\$647.56) and home (AU\$132.09) APAP titration. An intention-to-treat analysis confirmed the effectiveness of APAP titration compared with manual titration in the standard clinical setting.

Conclusions Among patients with moderate-severe OSA without serious co-morbidities, outcomes at 1 month indicate that APAP titration is more cost-effective than manual laboratory titration to determine an appropriate pressure for CPAP for long-term use; with the largest savings occurring in the home APAP patients.

Australian New Zealand Clinical Trials Registry Number ACTRN12608000054314.

\section{INTRODUCTION}

Obstructive sleep apnoea (OSA) is common, occurring to a clinically significant degree in $2-4 \%$ of the middle-aged population. ${ }^{1}$ OSA is associated with impaired quality of life and increased mortality from motor vehicle accidents ${ }^{2}$ and cardiovascular disease. ${ }^{3}$ There are a number of effective treatment options for OSA (including lifestyle advice and dental splint devices); however, the current standard of care for the majority of patients with moderate-severe OSA is nocturnal continuous positive airway pressure (CPAP). ${ }^{4} \mathrm{CPAP}$ is usually set at a 'fixed' pressure sufficient to prevent upper airway collapse in all body positions and sleep stages. The reference standard for determining a fixed pressure for CPAP is an attended overnight CPAP titration study with full polysomnographic (PSG) monitoring in a sleep laboratory (ie, manual titration). ${ }^{4}$ Manual titration, however, is time consuming and costly, and is an important factor delaying access to effective treatment.

Newer automatic positive airway pressure (APAP) devices are available which can control OSA by automatically adjusting pressure in response to device-measured physiological signals. The use of APAP titration to determine a fixed pressure for CPAP (which can subsequently be set on a standard CPAP machine) has the potential to reduce costs and improve access to treatment. A previous randomised controlled study ${ }^{5}$ validated the use of home APAP titration against manual titration on clinical outcomes for severe OSA (apnoea/hypoponea index (AHI) $\geq 30$, Epworth Sleepiness Score ${ }^{6}$ (ESS) $\geq 12$ ). In practice, the majority of patients with OSA treated with CPAP have moderate severity disease, but there are no data validating the use of APAP titration in patients with moderate-severe disease (AHI>15) against the reference standard of manual titration. Further, there is a paucity of randomised controlled data comparing costs of APAP titration with manual titration.

We hypothesised that APAP titration would be as effective as manual titration among patients with moderate-severe OSA. We chose average nightly CPAP use as the a priori primary outcome. A secondary objective was to determine direct costs of the methods.

\section{METHODS}

We undertook a parallel group randomised controlled trial comparing manual titration, attended laboratory APAP and home APAP titration on clinical outcomes in moderate-severe OSA.

\section{Patient selection}

The source population were consecutive patients who were referred to a tertiary hospital sleep service (Western Australian Sleep Disorders Research Institute, Sir Charles Gairdner Hospital, Perth, Western Australia), between March 2005 and December 2006, for possible OSA.

All patients underwent a comprehensive clinical assessment by an experienced sleep physician and a full overnight laboratory PSG. The physician recorded whether there was a history of, or 
treatment for, co-morbid conditions, for example hypertension or diabetes. The PSG was performed using the Compumedics E series (Compumedics Ltd, Abbotsford, Australia) which recorded the following signals: C3/A2 and C4/A1 EEG, bilateral electrooculograms, submental electromyogram (EMG), ECG, nasal and oronasal airflow (nasal pressure, thermistor), oximetry, chest and abdominal movement (inductance plethysmography), body position, sound intensity and bilateral tibial EMGs. Apnoeas and hypopnoeas were defined using standard criteria $^{7}$ and sleep architecture was scored according to the criteria of Rechtschaffen and Kales. ${ }^{8}$

Inclusion criteria were: symptoms of OSA, including sleepiness with an ESS $\geq 8$; AHI $\geq 15$ events/h slept; age between 17 and 85 years; living near the Sleep Service (usually $<50 \mathrm{~km}$ ); and no previous treatment for OSA. Exclusion criteria are shown in figure 1.

Ethical approval for the study was obtained from the local ethics committee, and informed consent was obtained from all participants. The trial was registered with the Australian New Zealand Clinical Trials Registry (ACTRN12608000054314).

\section{Protocol}

The protocol is detailed in figure 2. Eligible patients were told that three commonly used methods of determining a pressure for CPAP would be tested, either in the laboratory or in the home. They were not given information about the titration methods. Participants were allocated to a treatment arm using a random number generator and opaque envelopes.

On the evening prior to titration, all participants received $\sim 45 \mathrm{~min}$ of standardised education about OSA and CPAP treatment from a trained technologist blinded to treatment

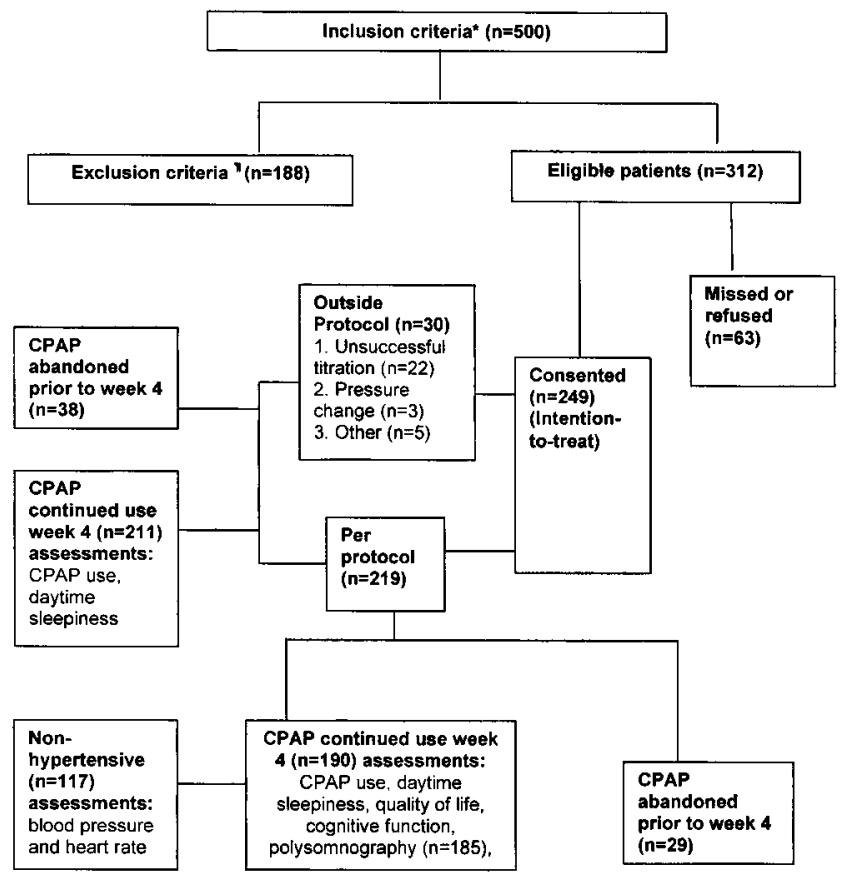

*Inclusion criteria (see text).

"Exclusion criteria: 1 . Body mass index $>45 \mathrm{~kg} / \mathrm{m}^{2} \quad(n=42) ; 2$. Significant lung disease including moderate-severe COPD or respiratory failure from any cause $(n=19)$; 3 . Significant cardiac disease, including cardiac failure with an ejection fraction $<45 \%$ or uncontrolled cardiac ischaemia $(n=13) ; 4$. Neuromuscular disease $(n=16) ; 5$. Previous stroke $(n=2) ; 6$. Predominant central sleep apnoea ( $>50 \%$ central apnoeas) $(n=4) ; 7$. Periodic leg movements $>15$ events/hr slept $(n=49) ; 8$. Severe medical illness or planned surgery $(n=11) ; 9$. Language impairment or psychiatric illness $(n=32)$.

Abbreviations: moderate-severe COPD: defined as physician diagnosis and airfow limitation that is not fully reversible' with FEV $1<70 \%$ predicted:
1. Celli B. R. and W. MacNee 2004. Standards for the diagnosis and treatment of patients with COPD: a summary of the
ATSIERS position paper. Eur. Respir.J. 23:932-946.

Figure 1 Patient flow diagram.

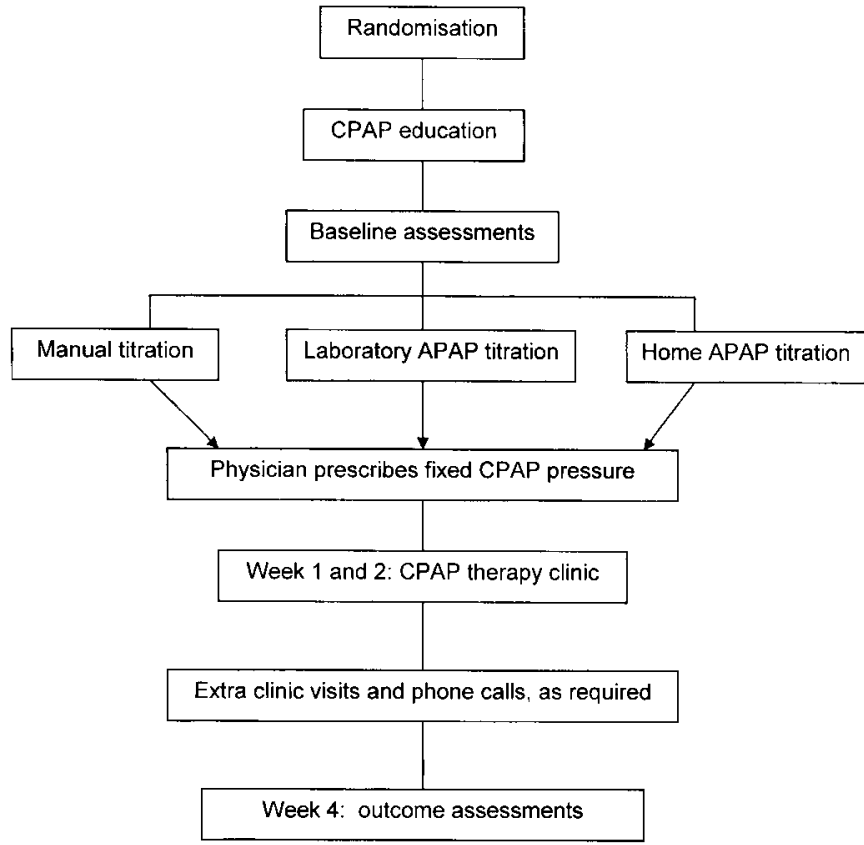

Figure 2 Protocol.

allocation. Potential side effects from CPAP and appropriate remedies were explained, and written information was provided. A CPAP interface was individually fitted from a wide range of interfaces to maximise comfort and minimise leak. All participants used an AutoSet Spirit S7 device (ResMed, Bella Vista, NSW, Australia) throughout the study. Participants were instructed not to discuss their titration with staff at subsequent study appointments.

Manual titration was performed with full PSG monitoring (see above) plus mask pressure, leak and flow signals. The pressure of CPAP was increased in $1-2 \mathrm{~cm} \mathrm{H}_{2} \mathrm{O}$ increments to abolish snoring, apnoeas, hypopnoeas and flow limitation, and to minimise EEG arousals in all stages of sleep and all sleep positions. CPAP was 'back-titrated' to allow for hysteresis. ${ }^{9} 10$

Laboratory APAP titration was undertaken without PSG monitoring but with real-time mask pressure, leak and flow monitoring in addition to video and audio signals. The overnight technologist was able to intervene for excessive mask leak (eg, by adjusting or changing the mask, or using a chin strap) or at the patient's request.

Home APAP patients were instructed to use the device that night at home and to return the following morning.

The morning after titration an experienced sleep physician (including $\mathrm{BS}, \mathrm{DH}, \mathrm{NMc}$ ) reviewed the titration data to determine a fixed pressure, blinded to participant information and, in the case of APAP titration, also blinded to the APAP group. Manual titration studies were analysed overnight and the physician determined a fixed pressure before the patient was discharged home in the morning. APAP titration data (laboratory and home) were downloaded using AutoScan 4.5 software (mask use, pressure and leak profile). A fixed pressure for APAP patients was determined, usually on the basis of the threshold at or below which pressure was maintained for $95 \%$ of the period of APAP application ('95th centile' pressure). Adjustments could be made to this determination to allow for pressure fluctuations associated with substantial leak ( $>241 / \mathrm{min})$ or mask removal.

A technologist reviewed the patient the morning following titration, to provide education about overnight treatment changes and to set a fixed pressure for CPAP. Any perceived 
problems arising during home APAP titration, such as mask fitting difficulties or CPAP side effects, were addressed.

Patients attended a combined sleep physician and technologist clinic review 1 and 2 weeks after titration. Additional clinic reviews were provided if needed. The physician, who was blinded to titration group and titration data, prescribed therapeutic interventions as clinically indicated. However, the pressure for CPAP could not be altered during the study from that determined from the titration methods described above, unless the physician deemed it clinically necessary. Participants were also provided with ready technologist phone contact for CPAP advice (daytime and answer machine after hours for morning reply).

Participants were considered to be per protocol (PP) (figure 1) unless: (a) the titration was 'unsuccessful' $(\mathrm{n}=22)$ (manual $(n=10)$; laboratory $\operatorname{APAP}(n=2)$; home APAP $(n=10)) ;(b)$ the physician changed the pressure for CPAP based on clinical judgement $(n=3)$; or $(c)$ other significant protocol violation $(\mathrm{n}=5)$ (physician withdrew patient (3), patient withdrew (1), inclusion error (1)).

Manual titration was considered 'unsuccessful' if there were $<4$ h of technically satisfactory data during sleep. APAP titration was considered 'unsuccessful' if the total recording time was $<5 \mathrm{~h}$ and/or median leak $>24 \mathrm{l} / \mathrm{min}$. In the home (but not the laboratory) APAP group if the first titration night was unsuccessful ( $n=32$ of 86 ) then a second attempt at successful titration was made the following night. When the home APAP patient was reviewed by the technologist the morning after their first unsuccessful night, potential problems were assessed and treated (eg, humidifier supplied for dry mouth) and the titration night was repeated.

Unsuccessful titrations in all groups were followed immediately by 1 week of home APAP titration and a fixed pressure was determined at week 1 clinic review, and clinical follow-up was unchanged from the above. Other patients outside the protocol were managed in a similar manner to PP; however, when patients were withdrawn, some review appointments were not according to the protocol schedule or they had additional titrations.

\section{Measurements}

\section{Baseline}

On the titration evening an ESS, SF36 (Short Form 36) quality of life questionnaire ${ }^{11}$ and Trails $\mathrm{A}$ and $\mathrm{B}$ cognitive function tests ${ }^{12}$ were measured. Resting blood pressure (BP) and heart rate (HR) were also measured according to current recommendations ${ }^{13}$ using a validated ${ }^{14}$ automated device (BPTru 100; BPTru Medical Devices, Coquitlam, BC, Canada) which calculated the average of five readings.

\section{PP patients}

\section{Week 4 outcomes}

The percentage of patients continuing to use CPAP at week 4 was determined. Assessments were made on the remaining PP patients continuing to use CPAP treatment, as follows: CPAP average nightly 'mask' use at prescribed pressure (AutoScan 5.4), ESS, SF36, Trails A and B, and evening BP and HR. These assessments were followed by an overnight laboratory PSG while the patient used CPAP at the prescribed pressure (the technologist could intervene only for technical problems with PSG signals). Week 4 PSG data were scored blinded to titration group and clinical information.

\section{CPAP interventions, staff time and other costs}

During the trial, CPAP interventions were recorded on participants. Hand-held stopwatches and electronic records (phone calls) were used to record the time clinical staff spent on patient management. Capital equipment and consumable costs per patient were estimated by crude amortisation over 5 years or the expected life of the consumable, respectively.

\section{Intention-to-treat (ITT) patients}

Core clinical outcomes (continued CPAP use, nightly CPAP use and ESS) were assessed. CPAP interventions, staff time and other costs were also assessed, as for PP patients.

\section{Statistical analysis}

Data were analysed using SPSS statistical software (SPSS for windows 15.0; Chicago, Illinois, USA). Statistical significance was considered to occur at $\mathrm{p}<0.05$.

The primary outcome was average nightly CPAP use for the first 4 weeks after titration. We calculated that 60 completed PP patients per group would be required to detect a minimum clinically significant difference of $1 \mathrm{~h}$ with a power of $80 \%$ at the 0.05 significance level (two sided), ${ }^{15}$ assuming an SD of 115 min. $^{16}$

Variables were described using means and SDs (parametric) and medians and IORs (non-parametric).

Comparisons between three independent variables were performed using one-way analysis of variance (ANOVA; parametric) or the Kruksal-Wallis test (non-parametric). Significant variables from these comparisons were further analysed using post hoc tests. $\chi^{2}$ tests or the Fisher exact test were used for categorical data.

\section{RESULTS}

Five hundred patients met the inclusion criteria and, of these, 188 were excluded for a variety of reasons (figure 1). Of the 312 eligible patients 63 either refused to consent, usually because of personal commitments, or were missed by the researchers.

\section{Baseline comparisons}

The 63 non-consented patients were similar to the 249 consented participants, and the titration groups were similar on baseline clinical data (table 1).

\section{Continuous positive airway pressure}

The mean CPAPs prescribed were similar in all PP groups: manual (11.2 \pm 2.3$)$, laboratory $(11.5 \pm 1.4)$ and home APAP $\left(10.9 \pm 1.3 \mathrm{~cm} \mathrm{H} \mathrm{H}_{2} \mathrm{O}, \mathrm{p}=0.08\right)$. In both the home and laboratory APAP groups the physician prescribed the 95th centile pressure $88 \%$ of the time, with no significant difference between the prescribed and 95 th centile pressures $(p>0.3)$. The mean CPAP pressures prescribed were also similar in ITT groups: manual $(11.3 \pm 2.3)$, laboratory $(11.5 \pm 1.4)$ and home APAP $(10.9 \pm 1.5 \mathrm{~cm}$ $\left.\mathrm{H}_{2} \mathrm{O}, \mathrm{p}=0.07\right)$.

\section{Week 4 outcomes \\ PP patients \\ CPAP compliance and clinical outcomes (see table $A$, online supplement)}

The number and percentage of patients continuing to use CPAP at week 4 was similar in all groups: manual $(n=62,87 \%)$, laboratory $(n=65,88 \%)$ and home $\operatorname{APAP}(n=63,85 \%, p=0.9)$. Among those continuing to use CPAP at week 4 there was no significant difference in average nightly use (primary outcome) among the groups; however, there was a trend to higher use in the laboratory APAP group $(5.15 \pm 1.9$, 95\% CI 4.68 to $5.62 \mathrm{~h} /$ night, $\mathrm{n}=65)$ compared with manual $(4.36 \pm 2.2,95 \%$ CI 3.81 to $4.91 \mathrm{~h} /$ night, $\mathrm{n}=62)$ and home APAP $(4.39 \pm 2.2,95 \%$ CI 3.83 to $4.94 \mathrm{~h} /$ night, $\mathrm{n}=61)$ groups $(\mathrm{p}=0.052)$. Baseline ESS was slightly higher in the 
Table 1 Comparison of baseline characteristics among titration groups (consented patients) and between consented and non-consented patients

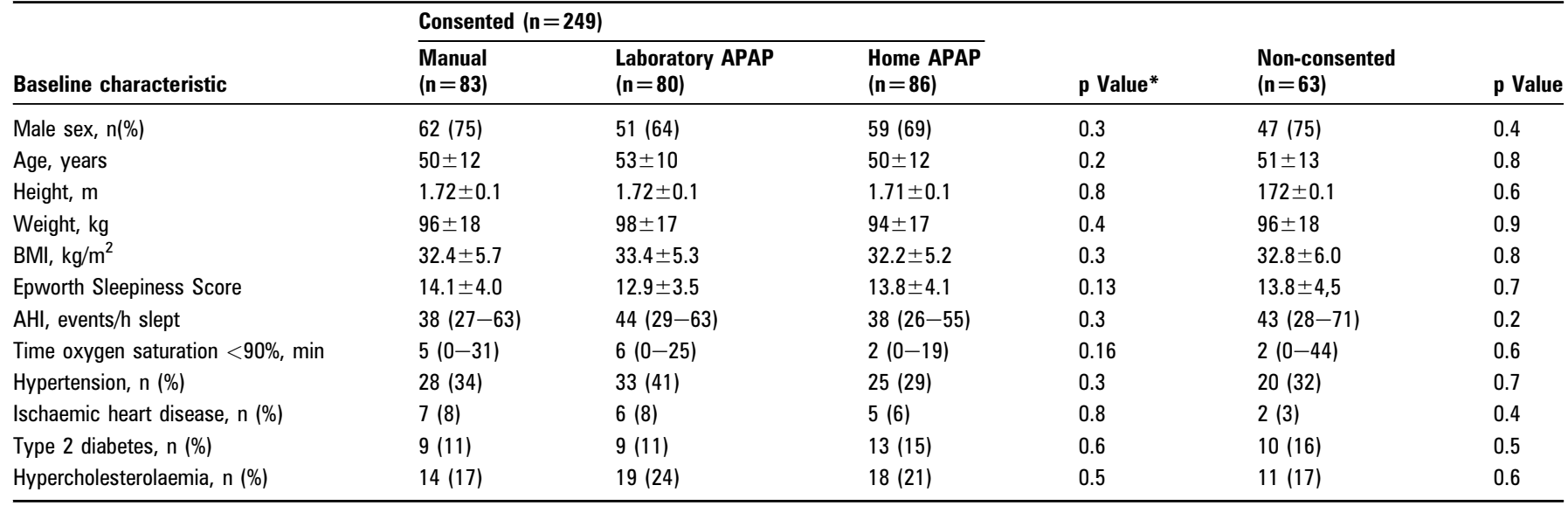

\footnotetext{
Data are presented as mean \pm SD, median (IOR) or number (\%).

Comparisons of consented with non-consented (refused or missed) patients.

*Comparisons of titration groups among consented patients.

AHI, apnoea+hypopnoea index; APAP, automatic positive airway pressure; BMI, body mass index.
}

manual (14.4 \pm 4.0$)$ compared with the laboratory APAP group $(12.6 \pm 3.1, p=0.025)$. Week 4 and change (week 4 minus baseline) in subjective sleepiness were similar in all groups. Quality of life and cognitive function showed closely similar outcomes in each group.

\section{Polysomnographic outcomes (table 2)}

Sleep architecture, control of OSA and CPAP device leak at week 4 were similar among the groups. Diagnostic PSG indices and change (week 4 minus diagnostic) in PSG indices were the same across groups (data not shown).

\section{Cardiovascular outcomes (see table $B$, on-line supplement)}

PP patients not diagnosed or treated for hypertension had similar baseline, week 4 and change (week 4 minus baseline) in systolic and diastolic BP. Baseline resting HR was similar among the groups. However, week $4 \mathrm{HR}$ was lower and there were signif-

Table 2 Comparison of week 4 per protocol overnight polysomnographic (PSG) outcomes on CPAP at the prescribed pressure

\begin{tabular}{|c|c|c|c|c|}
\hline $\begin{array}{l}\text { PSG outcome } \\
\text { (per protocol) }\end{array}$ & $\begin{array}{l}\text { Manual } \\
(n=59)\end{array}$ & $\begin{array}{l}\text { Laboratory } \\
\text { APAP } \\
(n=64)\end{array}$ & $\begin{array}{l}\text { Home } \\
\text { APAP } \\
(n=62)\end{array}$ & $\begin{array}{l}\text { p } \\
\text { Value }\end{array}$ \\
\hline Total recording time, min & $467 \pm 70$ & $457 \pm 71$ & $464 \pm 57$ & 0.7 \\
\hline Total sleep time, min & $351 \pm 79$ & $345 \pm 74$ & $364 \pm 71$ & 0.4 \\
\hline Stage $1+2, \min$ & $257 \pm 62$ & $248 \pm 59$ & $264 \pm 61$ & 0.3 \\
\hline Stage $3+4, \min$ & $34 \pm 32$ & $31 \pm 26$ & $32 \pm 22$ & 0.8 \\
\hline Stage REM, min & $63 \pm 35$ & $67 \pm 33$ & $71 \pm 31$ & 0.4 \\
\hline AHI, events/h slept & $7.1(3.9-16)$ & $6.7(4.1-14)$ & $8.0(5.2-14)$ & 0.8 \\
\hline $\begin{array}{l}\text { Arousal index, events } / \mathrm{h} \\
\text { slept }\end{array}$ & $23 \pm 12$ & $24 \pm 12$ & $22 \pm 9$ & 0.6 \\
\hline $\begin{array}{l}\text { 0xygen saturation time } \\
<90 \% \text {, min }\end{array}$ & $0(0-0)$ & $0(0-0)$ & $0(0-0)$ & 0.8 \\
\hline $\begin{array}{l}\text { Oxygen saturation } \\
\text { average, } \%\end{array}$ & $95 . \pm 82$ & $95.7 \pm 2$ & $96.0 \pm 2$ & 0.5 \\
\hline $\begin{array}{l}\text { Oxygen saturation } \\
\text { lowest, } \%\end{array}$ & $89.0 \pm 13$ & $88.2 \pm 12$ & $90.1 \pm 5$ & 0.6 \\
\hline $\begin{array}{l}\text { Median mask leak, } \\
\mathrm{l} / \mathrm{min}\end{array}$ & $3.6(0-10.8)$ & $1.2(0-8.1)$ & $1.2(0-6.0)$ & 0.16 \\
\hline $\begin{array}{l}\text { 95th centile mask leak, } \\
\text { l/min }\end{array}$ & $20.4(9-37.8)$ & $12.6(7.2-29.7)$ & $14.4(6-26.4)$ & 0.24 \\
\hline \multicolumn{5}{|c|}{$\begin{array}{l}\text { Data are presented as mean } \pm \text { SD or median (IQR). } \\
\text { CPAP use = average nightly 'mask on' time. } \\
\text { Five per protocol patients did not have overnight PSG at week } 4 \text { ( } 3 \text { manual, } 1 \text { laboratory } \\
\text { APAP, } 1 \text { home APAP titration). } \\
\text { AHI, apnoea/hypopnoea index; APAP, automatic positive airway pressure; } \\
\text { CPAP, continuous positive airway pressure; REM, rapid eye movement. }\end{array}$} \\
\hline
\end{tabular}

icant reductions (week 4 minus baseline) in HR in the laboratory $(-5.4 \pm 9.4 / \mathrm{min})$ compared with the home $(0.9 \pm 9.2 / \mathrm{min})$ APAP group ( $\mathrm{p}=0.008)$.

\section{CPAP interventions (see table $C$, on-line supplement)}

The home APAP patients were provided with fewer chin straps than the two laboratory groups, with a difference occurring on the titration night and not during the subsequent 4 weeks follow-up review. The home APAP group were also issued with more humidifiers than the laboratory APAP group.

\section{Costs}

Staff costs per patient were calculated using staff times and current pay scales, they were: manual, AU\$250.95; laboratory APAP, AU\$103.67; and home APAP, AU\$70.74. Time spent by clinical staff per patient in each group is shown in table 3 .

Capital equipment and consumable costs per patient were: manual, AU\$93.19; laboratory APAP, AU\$70.89; and home APAP, AU\$61.35. Current public hospital bed costs were AU\$473/ patient. Hence, total direct costs per patient were highest in manual (AU\$817.84), followed by laboratory APAP (AU\$647.56) and home APAP titration (AU\$132.09).

Table 3 Comparison of per protocol staff time per patient in the first 4 weeks of CPAP treatment

\begin{tabular}{lllll}
\hline $\begin{array}{l}\text { Staff time/patient } \\
\text { (minutes) }\end{array}$ & $\begin{array}{l}\text { Manual } \\
(\mathbf{n}=\mathbf{7 1})\end{array}$ & $\begin{array}{l}\text { Laboratory } \\
\text { APAP } \\
(\mathbf{n}=74)\end{array}$ & $\begin{array}{l}\text { Home } \\
\text { APAP } \\
(\mathbf{n}=74)\end{array}$ & p Value \\
\hline $\begin{array}{l}\text { Technologist } \\
\text { Education }\end{array}$ & $46.4 \pm 7.6$ & $42.6 \pm 6.0$ & $47.8 \pm 7.4$ & 0.4 \\
Titration & $142.0 \pm 49$ & $35.2 \pm 22$ & $\mathrm{~N} / \mathrm{A}$ & $<0.001$ \\
Titration study PSG scoring & $43.6 \pm 8.5$ & $\mathrm{~N} / \mathrm{A}$ & $\mathrm{N} / \mathrm{A}$ & $\mathrm{N} / \mathrm{A}$ \\
Titration morning & $10.1 \pm 6.8^{*}$ & $11.0 \pm 8.3 \dagger$ & $14.0 \pm 9.1^{*} \dagger$ & 0.02 \\
Follow-up clinics & $36.9 \pm 24.7$ & $35.1 \pm 23.3$ & $31.8 \pm 21.8$ & 0.4 \\
Phone calls & $1.9 \pm 4.9$ & $2.3 \pm 4.9$ & $0.7 \pm 2.6$ & 0.2 \\
Physician & & & & \\
Titration study reporting & $12.7 \pm 4.9 \neq$ & $1.8 \pm 6.7 \neq$ & $1.3 \pm 4.5 \neq$ & $<0.001$ \\
Follow-up clinics & $13.1 \pm 11.3$ & $12.8 \pm 9.6$ & $10.1 \pm 9.4$ & 0.15 \\
\hline
\end{tabular}

Phone calls, $60 \%$ consecutive sample; titration night, $60 \%$ consecutive sample; titration PSG study scoring, $40 \%$ sample; titration morning includes time to download data from device (APAP groups).

*Post hoc, LSD: $\mathrm{p}=0.01$.

†Post hoc, LSD: $p=0.04$

\#Post hoc, Tamhane: $p<0.001$.

APAP, automatic positive airway pressure; CPAP, continuous positive airway pressure; LSD, least significant difference; N/A, not applicable; PSG, polysmonography. 
The travel costs per patient (69 cents $/ \mathrm{km}$, Australian Taxation Office rates for a standard vehicle (1601-2000 cc engine)) were higher in the home APAP group (AU\$26.91 19.87) than the other groups (manual, AU\$15.04 \pm 10.28 ; laboratory APAP, AU $\$ 13.59 \pm 10.21$, both $\mathrm{p}<0.001)$.

\section{ITT patients CPAP compliance and ESS (see table D, on-line supplement)}

The number and percentage of patients continuing to use CPAP at week 4 were similar in all groups: manual $(n=71,86 \%)$, laboratory $(n=70,88 \%)$ and home APAP $(n=70,81 \%, p=0.5)$. Average nightly CPAP use was significantly higher in the laboratory APAP group $(5.15 \pm 1.9,95 \%$ CI $4.7-5.6 \mathrm{~h} /$ night, $\mathrm{n}=68)$ than in the other groups (manual, $4.38 \pm 2.2,95 \% \mathrm{CI}, 3.8$ to $4.9 \mathrm{~h} /$ night, $\mathrm{n}=70, \mathrm{p}=0.03$; home APAP, $4.24 \pm 2.2,95 \%$ CI 3.7 to $4.8 \mathrm{~h} /$ night, $\mathrm{n}=68, \mathrm{p}=0.01$ ). Week 4 and change (week 4 minus baseline) in subjective sleepiness (ESS) were similar in all groups (both $\mathrm{p} \geq 0.2$ ).

\section{CPAP interventions, staff time and other costs}

These were similar to PP outcomes (data not shown).

\section{DISCUSSION}

This is the first study to confirm the effectiveness and costeffectiveness of APAP titration against the reference standard of attended laboratory manual CPAP titration in moderate-severe OSA. We have shown that clinical outcomes from PP home and laboratory APAP titration are equivalent to those from manual titration, with lower cost and resource utilisation. Non-hypertensive laboratory APAP patients had a greater reduction in resting $\mathrm{HR}$ by week 4 than those titrated by home APAP, which may be related to a trend to higher nightly CPAP use in the laboratory APAP group. Technologists provided more chin straps and fewer humidifiers when titration occurred in the laboratory (ie, manual titration and laboratory APAP titration) compared with home APAP titration; however, interface leak, sleep architecture and control of OSA at week 4 were similar across the groups. An ITT analysis, which includes patients who were considered to have failed titration and others whose pressure was altered on clinical grounds during follow-up, confirms the effectiveness of APAP titration in the standard clinical setting.

The primary aim of this study is to assess whether the newer (APAP) titration methods are associated with clinical outcomes that are non-inferior to the standard methods while requiring less resources and cost. To establish non-inferiority of the newer methods the most important outcome is the PP outcome since an ITT analysis, the preferred analysis for a placebo control trial, tends to be conservative and is less likely to show an inferiority of the newer methods. By the same reasoning we chose not to include clinical outcome assessments of those patients who abandoned CPAP by week 4, since this will also tend to minimise differences between the groups. Costs, however, have been calculated up to week 4 or until CPAP is abandoned prior to week 4.

Intensive nurse-led support, in the first months of treatment, has been shown to improve CPAP compliance, ${ }^{17}$ and a retrospective study suggests therapist support provided by laboratory titration is associated with improved CPAP compliance. ${ }^{18}$ Hence, we were concerned that APAP titration, particularly in the home setting, may result in reduced compliance due to lack of immediate therapist support and/or reduced therapist intervention on the titration night. For this reason we chose average nightly CPAP use as the a priori primary outcome. The only other randomised controlled trial comparing home APAP with manual titration recruited patients with severe disease. ${ }^{5} \mathrm{We}$ extend those previous findings ${ }^{5}$ by showing that, compared with manual titration, both home and laboratory APAP titration lead to similar CPAP use and equivalent clinical outcomes after 4 weeks for patients with moderate--severe disease.

We are aware of only one other study comparing laboratory APAP with home APAP $^{19}$ for titration, which showed equivalent clinical outcomes but made no assessment of cardiovascular changes. Further, the current study is the first to show equivalent clinical outcomes from laboratory APAP compared with the gold standard of manual titration. The laboratory APAP group in our study showed a trend (PP) or significant (ITT) highest nightly CPAP use at week 4 , with $\sim 45$ min extra use compared with the other groups. The clinical significance of this is uncertain and we found no differences in clinical outcomes between the groups, as expected based on our power calculation assumption of $1 \mathrm{~h}$ difference in CPAP use needed to detect clinical benefit. However, a small difference in resting $\mathrm{HR}$ at week 4 (and reduction in HR) was associated with laboratory APAP titration, compared with home APAP titration. The difference may be due to higher nightly CPAP use associated with laboratory APAP titration leading to a greater net reduction of sympathetic activity. ${ }^{20}$ This finding may be important if the $\mathrm{HR}$ reduction persists long term since $\mathrm{HR}$ is associated with cardiovascular morbidity and mortality. ${ }^{21}$

The current study is the first to compare the pattern of CPAP interventions associated with (laboratory) manual titration, laboratory APAP and home APAP titration. Both laboratory groups were issued more chin straps, presumably due to therapists responding in real time to mask leaks. Despite this differing pattern of chin strap use, there was no significant difference in mask leak, sleep architecture or control of OSA between the laboratory groups and the home APAP group at week 4 . Increased humidifier provision in the home compared with the laboratory APAP group may reflect the lower chin strap provision.

We are aware of only one small $(n=35)$ randomised controlled study, among subjects with severe OSA (AHI $\geq 30),{ }^{22}$ indicating the cost-effectiveness of home APAP titration compared with manual titration and one study showing that home APAP is cheaper than laboratory APAP titration. ${ }^{19}$ Our large study, including moderate and severe disease, confirms the costeffectiveness of APAP titration compared with manual titration. By calculating the direct costs associated with titration for patients attending a tertiary sleep centre we have demonstrated the large potential savings resulting from home APAP titration and smaller savings from laboratory APAP titration compared with manual titration.

In the standard clinical setting, some titrations may not be considered satisfactory due, for example, to inadequate duration of the patient's sleep (manual titrations) or the study length (APAP titrations). We used conservative, definitions for titration success. Further, we allowed a maximum of two nights for successful home APAP titration since we did not wish to confound the comparison of methods of titration with potential CPAP-induced changes in pressure requirements over time. There is a paucity of data to guide decisions on how to manage those who 'fail' titration. We decided to use a week of home APAP to manage titration failures as this provided a consistent approach and did not alter our follow-up protocol. The ITT analysis on core clinical outcomes confirms that the strategies we used to manage unsuccessful titrations are effective.

The results of the current study are applicable to the ResMed AutoSet Spirit and it cannot be concluded that other automatic 
CPAP devices would provide the same results. ${ }^{4}$ Further, our results apply after careful selection of patients for APAP by excluding those with severe co-morbidities ${ }^{4}$ and by providing close clinical follow-up in the first few weeks of CPAP use. ${ }^{4}$ We chose a conservative approach by excluding those with a body mass index $>45 \mathrm{~kg} / \mathrm{m}^{2}$ in view of the increased risk of hypoventilation associated with gross obesity. Similarly, we excluded those with periodic limb movements (PLMs) $>15$ events/h slept as it is unclear whether the co-existence of significant PLMs would be affected by the titration method, particularly since we planned to include patients with moderately severe OSA. The current study assesses short-term outcomes; however, shortterm CPAP use is strongly predictive of long-term use. ${ }^{23} 24$

In conclusion, in carefully selected patients with moderate-severe OSA APAP titration is more cost-effective than manual laboratory titration to determine an appropriate pressure for CPAP for long-term use, with the largest savings occurring where APAP titration is performed at home.

Acknowledgements This study could not have been conducted without the help of the study participants, technologists, administrative support and physicians at the Western Australian Sleep Disorders Research Institute. We thank Dr Stewart Cullen for assistance with sleep study reporting. We thank Michael Phillips, Biostatistician at the Western Australian Institute for Medical Research, for his assistance at the design, analysis and interpretation stages of the study.

Funding ResMed Ltd, Australia. The funding source played no role in the study design, the analysis or interpretation of data or the decision to submit the manuscript for publication.

Competing interests DRH has served as a consultant to ResMed Ltd, Inspiration medical and Apnex medical. NMc received \$AU54 000 In 2005 and 2006 from ResMed Ltd as a grant to help fund the current study.

Ethics approval This study was conducted with the approval of the ethics committee of the Sir Charles Gairdner Hospital, Perth, Australia.

Provenance and peer review Not commissioned; externally peer reviewed.

\section{REFERENCES}

1. Young T, Palta M, Dempsey J, et al. The occurence of sleep-disordered breathing among middle-aged adults. N Engl J Med 1993;328:1230-5.

2. Ellen RL, Marshall SC, Palayew M, et al. Systematic review of motor vehicle crash risk in persons with sleep apnea. J Clin Sleep Med 2006:2:193-200.

3. Marin JM, Carrizo SJ, Vicente E, et al. Long-term cardiovascular outcomes in men with obstructive sleep apnoea-hypopnoea with or without treatment with continuous positive airway pressure: an observational study. Lancet 2005;365:1046-53.
4. Morgenthaler TI, Aurora RN, Brown T, et al. Practice parameters for the use of autotitrating continuous positive airway pressure devices for titrating pressures and treating adult patients with obstructive sleep apnea syndrome: an update for 2007. An American Academy of Sleep Medicine report. Sleep 2008:31:141-7.

5. Masa JF, Jimenez A, Duran J, et al. Alternative methods of titrating continuous positive airway pressure: a large multicenter study. Am J Respir Crit Care Med 2004;170:1218-24

6. Johns MW. A new method for measuring daytime sleepiness: the Epworth sleepiness scale. Sleep 1991:14:540-5.

7. Sleep-related breathing disorders in adults: recommendations for syndrome definition and measurement techniques in clinical research. The Report of an American Academy of Sleep Medicine Task Force. Sleep 1999:22:667-89.

8. Rechtschaffen A, Kales A. A manual of standardized terminology and scoring system for sleep stages of human subjects. Washinton, DC: U.S.government printing office, NIH Publication No.204, 1968.

9. Montserrat JM, Ballester E, Olivi H, et al. Time-course of stepwise CPAP titration. Behavior of respiratory and neurological variables. Am J Respir Crit Care Med 1995:152:1854-9.

10. Condos R, Norman RG, Krishnasamy I, et al. Flow limitation as a noninvasive assessment of residual upper-airway resistance during continuous positive airway pressure therapy of obstructive sleep apnea. Am J Respir Crit Care Med 1994; 150:475-80.

11. Jenkinson C, Coulter A, Wright L. Short form 36 (SF 36) health survey questionnaire: normative data for adults of working age. BMJ 1993;306:1437-40.

12. Reitan RM. Validity of the trail making test as an indicator or organic brain damage Percept Motor Skills 1958:8:271-6.

13. The sixth report of the Joint National Committee on prevention, detection, evaluation and treatment of high blood pressure. Arch Intern Med 1997:157:2413-46.

14. Mattu GS, Heran BS, Wright JM. Overall accuracy of the BpTRU - an automated electronic blood pressure device. Blood Press Monit 2004;9:47-52.

15. Altman DG. Statistics and ethics in medical research: III How large a sample? BM 1980;281:1336-8.

16. Massie CA, McArdle N, Hart RW, et al. Comparison between automatic and fixed positive airway pressure therapy in the home. Am J Respir Crit Care Med 2003;167:20-3.

17. Hoy CJ, Vennelle M, Kingshott RN, et al. Can intensive support improve continuous positive airway pressure use in patients with the sleep apnea/hypopnea syndrome? Am J Respir Crit Care Med 1999;159:1096-100.

18. Means MK, Edinger JD, Husain AM. CPAP compliance in sleep apnea patients with and without laboratory CPAP titration. Sleep Breath 2004;8:7-14.

19. Cross MD, Vennelle M, Engleman HM, et al. Comparison of CPAP titration at home or the sleep laboratory in the sleep apnea hypopnea syndrome. Sleep 2006:29:1451-5.

20. Coy TV, Dimsdale JE, Ancoli-Israel S, et al. Sleep apnoea and sympathetic nervous system activity: a review. J Sleep Res 1996:5:42-50.

21. Cook S, Togni M, Schaub MC, et al. High heart rate: a cardiovascular risk factor? Eur Heart J 2006;27:2387-93.

22. Planes C, D'Ortho MP, Foucher A, et al. Efficacy and cost of home-initiated autonCPAP versus conventional nCPAP. Sleep 2003:26:156-60.

23. McArdle N, Devereux $\mathrm{G}$, Heidarnejad $\mathrm{H}$, et al. Long-term use of CPAP therapy for sleep apnea/hypopnea syndrome. Am J Respir Crit Care Med 1999;159:1108-14

24. Kribbs NB, Pack Al, Kline LR, et al. Objective measurement of patterns of nasal CPAP use by patients with obstructive sleep apnea. Am Rev Respir Dis 1993; 147:887-95. 